\title{
Association between Enamel Hypoplasia and Dental Caries in Primary Second Molars: A Cohort Study
}

\author{
L. Hong ${ }^{a} \quad$ S.M. Levy ${ }^{b, c} \quad$ J.J. Warren ${ }^{b} \quad$ B. Broffitt ${ }^{b}$ \\ a Department of Dental Public Health and Behavioral Science, School of Dentistry, University of Missouri-

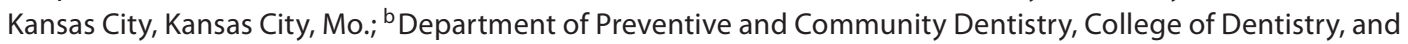 \\ 'Department of Epidemiology, College of Public Health, University of lowa, lowa City, lowa, USA
}

\section{Key Words}

Dental caries • Enamel hypoplasia • Primary teeth

\begin{abstract}
The purpose of this study was to assess the longitudinal relationships between enamel hypoplasia and caries experience of primary second molars. The study sample was 491 subjects who received dental examinations at both age 5 and 9 by the calibrated examiners. Four primary second molars ( $n=1,892$ ) were scored for the presence of enamel hypoplasia for each participant. Caries presence and number of decayed and filled surfaces (dfs) were determined at age 5 and 9. The relationships between enamel hypoplasia and caries experience were assessed. Among primary second molars, $3.9 \%$ of children and $1.7 \%$ of primary second molars had enamel hypoplasia. At age 5, 36.8\% of children with hypoplasia had caries, while $16.9 \%$ of children without enamel hypoplasia had caries. At age 9, the corresponding numbers were $52.6 \%$ for children with hypoplasia and $34.5 \%$ for children without hypoplasia, respectively. At the tooth level, for age $5,28.1 \%$ of teeth with hypoplasia had caries (mean dfs = 0.40 ), and $7.6 \%$ of teeth without hypoplasia had caries (mean $\mathrm{dfs}=0.11)$. At age 9 , the corresponding numbers were $41.9 \%$ (mean dfs $=0.76$ ) for teeth with hypoplasia and 18.3\% (mean $\mathrm{dfs}=0.34$ ) for teeth without hypoplasia. In multivariable logistic regression analyses, teeth of subjects with enamel hy-
\end{abstract}

poplasia had a significantly higher risk for caries at age 5 and 9 after controlling for other risk factors. Enamel hypoplasia appears to be a significant risk factor for caries and should be considered in caries risk assessment.

Copyright $\odot 2009$ S. Karger AG, Basel

Developmental enamel defects are disturbances during enamel formation and may be manifested as enamel hypoplasia or opacities. Enamel hypoplasia is a quantitative defect associated with reduced thickness of enamel formed during the secretory stage of amelogenesis [Suckling, 1989; Seow, 1991]. Developmental enamel defects, such as hypoplasia, have been speculated to increase the risk of dental caries in the affected teeth [Pascoe and Seow, 1994; Li et al., 1996; Lai et al., 1997]. Defective enamel sites (hypoplasia or hypocalcification) may provide suitable local environment for adhesion and colonization of cariogenic bacteria, and bacteria may retain at the base of the defect in contact with exposed dentin, thus dental caries on these defective sites may develop more rapidly [Li et al., 1996]. Defective enamel has higher acid solubility than normal enamel and is more susceptible to caries attack [Zheng et al., 1998]. The association between enamel hypoplasia and dental caries has been reported in several cross-sectional studies [Matee et al., 1994; Pascoe and Seow, 1994; Li et al., 1996; Ellwood and O’Mullane, 1996;

\section{KARGER}

Fax +41613061234 E-Mail karger@karger.ch www.karger.com
(C) 2009 S. Karger AG, Basel

0008-6568/09/0435-0345\$26.00/0

Accessible online at:

www.karger.com/cre
Liang Hong

Department of Dental Public Health, 396A School of Dentistry

University of Missouri-Kansas City, 650 East 25th Street

Kansas City, MO 64108-2784 (USA)

Tel. +1 816235 6745, Fax +1 816235 5472, E-Mail hongli@umkc.edu 
Kanchanakamol et al., 1996; Milgrom et al., 2000; Montero et al., 2003; Daneshkazemi and Davari, 2005] and two longitudinal studies [Lai et al., 1997; Oliveira et al., 2006]. Enamel defects such as enamel hypoplasia appear to be a significant, reliable risk marker or predictor for dental caries; however, most of these studies were cross-sectional and did not account for other important factors, such as fluoride exposure. The duration of the two longitudinal studies collecting caries data was 36 and 52 months, respectively. Therefore, a well-designed, prospective, longitudinal study, which can take multiple important factors into consideration, is needed.

The Iowa Fluoride Study (IFS) is a longitudinal study of a cohort (from birth) concerning the relationships among fluoride exposures, biological and environmental factors, and oral health. Using data collected in the IFS on dental caries and enamel hypoplasia for primary teeth and information on important covariates, this article reports on associations between enamel hypoplasia and dental caries of primary second molars at approximately 9 years of age.

\section{Subjects and Methods}

\section{Subjects}

Children were participants in the IFS [Levy et al., 1997, 1998; Hong et al., 2004]. Newborn infants were recruited at birth between March 1992 and February 1995 from 8 Iowa hospitals, and a total of 1,390 children participated in the study. The Institutional Review Board at the University of Iowa approved all components of the IFS, and written informed consent was obtained from mothers at recruitment and again at the time of examinations. Demographic characteristics at baseline were collected and previously described [Levy et al., 1997, 1998]. Briefly, this cohort is predominantly Caucasian (about 98\%), 51\% girls, from families of relatively high socioeconomic status (SES, $71 \%$ having a family income of USD 30,000 or more and $46 \%$ of mothers having completed 4 years of college), $44 \%$ were first-born, 32\% had been breast-fed for at least 6 months, only $4 \%$ had low birth weight and $3 \%$ had developmental disorders [Hong et al., 2004]. There were 698 subjects who received dental examinations of the primary dentition at age 5, and 603 who received second dental examinations at age 9 . This report concerns a sample of 491 subjects who had participated in both dental exams at age 5 and 9 and had baseline demographic information and water fluoride assessments.

\section{Data Collection}

Parents were mailed the IFS questionnaires at 3- to 6-month intervals including a series of items concerning children's fluoride exposures and ingestion from various sources during the preceding time period or weeks. Information regarding the children's beverage intakes, breast-feeding patterns, general health/illnesses, and oral health behaviors was also obtained. Combined fluoride concentrations in water at home, school and childcare were individually determined for each subject from public, private well, and/or bottled water sources. Daily fluoride intake (mg) was estimated from water, beverages and selected foods, dietary fluoride supplements, and fluoride dentifrice based on parents' responses to the series of questions. Parents' responses were not validated, but reliability was assessed for selected questions [Levy et al., 1997, 1998].

\section{Dental Examinations}

Dental examinations, including examination of hypoplasia and dental caries, were conducted using portable chair and halogen head light (DenLite; Welch-Allyn Medical Products, Inc., Skaneateles, N.Y., USA) at the General Clinical Research Center at the University of Iowa, or at one of several community locations by trained and calibrated dentist examiners using standardized, portable dental equipment [Slayton et al., 2001; Warren et al., 2002]. Teeth were evaluated for the presence of enamel hypoplasia using a mouth mirror and exam light, but without drying the teeth. Presence or absence of enamel hypoplasia was recorded separately for each tooth. Deficiency in enamel formation such as pits and linear grooves was recorded as enamel hypoplasia, while localized opacities that were white, yellow, or brownish in color were recorded as demarcated opacities. Enamel hypoplasia was differentiated from fluorosis [Pendrys, 1990] and other nonfluoride enamel defects using the criteria of Russell [1961]. Nonfluoride opacities are most commonly creamy-yellow to brown in color, well demarcated, and on the smooth surfaces. Fluorosis is more symmetrical and more diffuse, with white striations or patches that do not have well-defined margins [Slayton et al., 2001].

\section{Children's Dental Caries Experience}

For the dental caries examinations, halogen headlights were used, teeth were dried, and the DenLite mirror system provided additional lighting to augment the primarily visual examination, but a dental explorer was used to confirm questionable caries findings. A modified $\mathrm{D}_{1}-\mathrm{D}_{3}$ version of the criteria of Pitts and Fyffe [1988], Pitts [1997] and Ismail et al. [1992] was used to record caries lesions in this study. Our criteria [Warren et al., 2002] combined cavitated enamel $\left(\mathrm{d}_{2}\right)$ and dentin $\left(\mathrm{d}_{3}\right)$ lesions into a single $d_{2-3}$ category that was distinguished from $d_{1}$ (noncavitated) lesions. For this report, caries experience was considered in two ways. First, caries presence was defined as having any decayed (cavitated $\mathrm{d}_{2-3}$ lesions) and/or filled surfaces on primary second molars (yes/no). Second, the number of decayed (cavitated $d_{2-3}$ lesions) and/or filled surfaces (dfs) was calculated on primary second molars. These caries measures were calculated for both age 5 and 9 exams. Caries incidence was computed as the percentage of subjects with new caries, and caries increments as increases in $d_{2-3} f s ; d_{1}$ lesions were not used for these analyses. Teeth with a dental sealant were excluded from all analysis.

All examinations were done by one of three trained and calibrated dentist examiners, with calibration sessions scheduled before and midway through each set of exams. A small percentage of subjects was used for duplicate examinations to assess interexaminer reliability. All teeth were examined for both hypoplasia and caries, but only primary second molars were used for these analyses, since they were most consistently present for both examinations at age 5 and 9. Person-level hypoplasia and caries were defined as present whenever any of the primary second molars exhibited hypoplasia or caries. 
Statistical Analysis

Descriptive statistics were generated for caries experience by enamel hypoplasia status. Bivariate analysis was conducted at both the person and tooth levels. At the person level, children were classified into two mutually exclusive groups: with and without hypoplasia. Using the no-hypoplasia group as the reference, caries in children with enamel hypoplasia (both prevalence and $\mathrm{d}_{2-3} \mathrm{fs}$ ) was compared at age 5 and 9 using the $\chi^{2}$ test for prevalence and the Wilcoxon two-sample test for $\mathrm{d}_{2-3} \mathrm{fs}$. Caries increase from age 5 to 9 (percent incidence and $\mathrm{d}_{2-3}$ fs increment) was also compared. Bivariate analyses at the tooth level were conducted using generalized log-linear models (to compensate for intrasubject correlations) for caries presence and generalized linear models with negative binomial distribution for $\mathrm{d}_{2-3} \mathrm{fs}$.

Multivariable regression models were developed for caries at the tooth level using enamel hypoplasia as the explanatory factor, after controlling for other potentially important factors. These other potential predictors included gender, childhood illness (yes/ no), gestational weeks, birth weight, breast-feeding for 6 months or more (yes/no), SES [Shenkin et al., 2004], fluoride concentration of home drinking water (tap, well, and/or bottled), average daily fluoride intake, average daily soda pop intake, daily toothbrushing frequency, and previous (age 5) $\mathrm{d}_{2-3}$ fs (for age 5-9 increment models only). Three levels of SES were defined: (1) low families with less than USD 30,000 income per year and in which mothers did not have a 4-year college degree; (2) middle - families with an annual income of USD 30,000-49,999, but excluding those with mothers having a graduate or professional degree, or less than USD 30,000 but having mothers with a 4-year college or graduate/professional degree; (3) high - mothers with a graduate/ professional degree and USD 30,000 or more annual family income or USD 50,000 or more income regardless of the mother's educational level.

For these other potential predictors, bivariate associations with caries at age 5 and 9 were assessed using simple logistic regression for prevalence data (percentage of subjects with caries) and Spearman correlations for count data $\left(\mathrm{d}_{2-3} \mathrm{fs}\right)$ at the person level (not tooth level). Those variables that were significant at $\alpha=$ 0.10 were used in backward elimination multivariable models, and the final model included variables that were jointly significant at $\alpha=0.05$, plus hypoplasia. Because caries data for individual teeth within the same subject are correlated, statistical methods must account for the correlation between teeth within a subject. A subject-level hypoplasia score was defined as the mean number of primary second molars with hypoplasia and was used to assess between-subject effects of hypoplasia. A within-subject hypoplasia score for each tooth was also defined using contrasts between tooth-level scores (0.1) and person-level averages. For example, a subject having one tooth with hypoplasia (out of 4) would have four within-subject hypoplasia scores $(0.75,-0.25,-0.25$, and -0.25$)$. Use of the within-subject hypoplasia score in regression models gives insight into differences between hypoplastic teeth vs. nonhypoplastic teeth within the same subject [Mancl et al., 2000]. Logistic generalized estimating equation models (GEE) [Evans and Li, 2005] were used for binary outcomes (yes/no), and negative binomial GEE models for count outcomes $\left(\mathrm{d}_{2-3} \mathrm{fs}\right)$. All statistical tests were conducted using SAS 9.1 for Windows, with $\mathrm{p}$ values below 0.05 being considered as statistically significant.

\section{Results}

Interexaminer reliability was assessed from duplicate examinations. Weighted kappa for person-level $\mathrm{d}_{2-3} \mathrm{fs}$ was 0.90 at age 5 and 0.90 at age 9 . For enamel hypoplasia, person-level kappa was 1.0 at age 5 .

Considering the primary second molars of 491 children, $3.9 \%$ of children had hypoplasia, $17.7 \%$ of children had caries at age 5, 35.2\% had caries at age 9 , and $31.1 \%$ developed new carious lesions from age 5 to 9. Table 1 shows that at the tooth level, $1.7 \%$ of all primary second molars had hypoplasia. Among all 1,892 primary second molars included in the analyses, $7.9 \%$ had caries at age 5 (mean $\mathrm{d}_{2-3} \mathrm{fs}=0.11$ per tooth), $18.7 \%$ at age $9\left(\right.$ mean $\mathrm{d}_{2-3} \mathrm{fs}$ $=0.34$ per tooth), and $14.5 \%$ had an increase in caries from age 5 to 9 . Caries distributions for individual molars (A, J, K, T: FDI 55, 65, 75, 85) are also included in table 1 .

The bivariate relationships between enamel hypoplasia (age 5 exam) and caries experience of primary second molars at ages 5 and 9 , and incidence from age 5 to 9 are presented in table 2. Children with enamel hypoplasia had more caries than those without enamel hypoplasia for both prevalence/presence and $\mathrm{d}_{2-3}$ fs at both ages 5 and 9 at both the person and tooth levels. The bivariate association was statistically significant for caries at age 5 for both person and tooth levels, even though there were only 19 children with hypoplasia. For example, at the person level, $36.8 \%$ of children with hypoplasia had caries at age 5 vs. only $16.9 \%$ of children without enamel hypoplasia (relative risk, $\mathrm{RR}=2.17$ ). With larger sample sizes in the tooth level analyses, teeth with enamel hypoplasia had significantly more caries at age $9(\mathrm{RR}=1.75, \mathrm{p}<0.01)$. However, while caries increment from age 5 to 9 was elevated for subjects with hypoplasia, caries was not significantly related to enamel hypoplasia at either the person or tooth level ( $R R=1.55$ for each).

Screening for other important potential predictors for caries levels (yes/no at age 5, age 9 and age 5-9 increment) used simple logistic regression (at the person level) and examined bivariate associations with gender, age, childhood illness, gestational age, birth weight, breast-feeding ( $<6$ months vs. $\geq 6$ months), SES (three levels), fluoride concentration of home drinking water, average daily fluoride intake (mg), average daily soda pop intake (oz) and daily toothbrushing frequency. Spearman correlations were used to examine the bivariate associations of these same potential risk factors with caries counts $\left(\mathrm{d}_{2-3} \mathrm{fs}\right)$ for age 5, age 9 and age 5-9 increments. Variables having associations with $\mathrm{p}<0.10$ are listed in the foot- 
Table 1. Distribution of enamel hypoplasia, dental caries in primary second molars and subject-level factors

\begin{tabular}{|c|c|c|c|c|c|}
\hline & $\begin{array}{l}\text { Tooth A } \\
\text { (FDI 55) }\end{array}$ & $\begin{array}{l}\text { Tooth J } \\
\text { (FDI 65) }\end{array}$ & $\begin{array}{l}\text { Tooth K } \\
\text { (FDI 75) }\end{array}$ & $\begin{array}{l}\text { Tooth T } \\
\text { (FDI 85) }\end{array}$ & $\begin{array}{l}\text { All second molars } \\
(\mathrm{n}=1,892)^{\mathrm{a}}\end{array}$ \\
\hline Enamel hypoplasia prevalence, \% & 1.5 & 1.1 & 2.1 & 2.1 & 1.7 \\
\hline \multicolumn{6}{|l|}{$d_{2-3} f s$} \\
\hline Age 5 & 8.4 & 7.2 & 9.1 & 7.0 & 7.9 \\
\hline Age 9 & 20.4 & 19.4 & 17.3 & 17.7 & 18.7 \\
\hline \multicolumn{6}{|l|}{ Incidence, $\%$} \\
\hline Age $5-9$ & 16.2 & 16.0 & 13.0 & 13.0 & 14.5 \\
\hline \multicolumn{6}{|l|}{ Incidence, mean $\pm S D$} \\
\hline Age 5 & $0.11 \pm 0.38$ & $0.10 \pm 0.42$ & $0.11 \pm 0.38$ & $0.10 \pm 0.42$ & $0.11 \pm 0.40$ \\
\hline Age 9 & $0.39 \pm 0.86$ & $0.37 \pm 0.83$ & $0.32 \pm 0.84$ & $0.28 \pm 0.71$ & $0.34 \pm 0.82$ \\
\hline Increment & $0.29 \pm 0.74$ & $0.28 \pm 0.71$ & $0.22 \pm 0.67$ & $0.19 \pm 0.56$ & $0.25 \pm 0.67$ \\
\hline \multirow[t]{2}{*}{ Subject-level factor } & \multicolumn{3}{|l|}{ Prevalence, \% } & \multirow[t]{2}{*}{$\mathrm{p}$ value $^{\mathrm{b}}$} & \\
\hline & $\begin{array}{l}\text { subjects without } \\
\text { hypoplasia }\end{array}$ & $\begin{array}{l}\text { subjec } \\
\text { hypor }\end{array}$ & & & \\
\hline \multicolumn{6}{|l|}{ Gender } \\
\hline Boys & 49 & \multicolumn{2}{|l|}{53} & \multicolumn{2}{|l|}{0.76} \\
\hline Girls & 51 & 47 & & & \\
\hline \multicolumn{6}{|l|}{ SES } \\
\hline Low & 22 & \multicolumn{2}{|l|}{6} & \multicolumn{2}{|l|}{0.13} \\
\hline Middle & 36 & \multicolumn{2}{|l|}{56} & & \\
\hline High & 43 & \multicolumn{2}{|l|}{39} & \\
\hline Childhood illness & 25 & \multicolumn{2}{|l|}{37} & \multicolumn{2}{|l|}{0.25} \\
\hline \multirow[t]{2}{*}{ Breast-fed 6 months or more } & 32 & \multicolumn{2}{|l|}{39} & \multicolumn{2}{|l|}{0.52} \\
\hline & mean $\pm \mathrm{SD}$ & \multicolumn{2}{|c|}{ mean $\pm \mathrm{SD}$} & \multicolumn{2}{|l|}{$\mathrm{p}$ value $\mathrm{c}^{\mathrm{c}}$} \\
\hline Gestational weeks & $39.5 \pm 2.1$ & $40.3 \pm$ & & 0.01 & \\
\hline Birth weight, kg & $3.5 \pm 0.6$ & $3.6 \pm$ & & 0.15 & \\
\hline \multicolumn{6}{|l|}{ Dental exam age, years } \\
\hline Primary dentition exam 1 & $5.1 \pm 0.4$ & \multicolumn{2}{|c|}{$5.1 \pm 0.3$} & \multicolumn{2}{|l|}{0.56} \\
\hline Mixed dentition exam 2 & $9.2 \pm 0.7$ & $9.2 \pm$ & & 0.95 & \\
\hline Age increment (exam 1-2) & $4.1 \pm 0.7$ & $4.2 \pm$ & & 0.71 & \\
\hline Home tap fluoride, ppm & & & & & \\
\hline Age 3-5 & $0.83 \pm 0.45$ & $0.67 \pm$ & & 0.11 & \\
\hline Age 6-9 & $0.81 \pm 0.43$ & $0.64 \pm$ & & 0.09 & \\
\hline Daily fluoride intake, mg & & & & & \\
\hline Age 3-5 & $0.77 \pm 0.37$ & $0.72 \pm$ & & 0.55 & \\
\hline Age 6-9 & $0.72 \pm 0.35$ & $0.56 \pm$ & & 0.43 & \\
\hline Daily soda pop intake, oz & & & & & \\
\hline Age 3-5 & $2.2 \pm 2.1$ & $1.7 \pm$ & & 0.32 & \\
\hline Age 6-9 & $3.0 \pm 2.4$ & $2.9 \pm$ & & 0.87 & \\
\hline Daily toothbrushing frequency & & & & & \\
\hline Age $3-5$ & $1.3 \pm 0.5$ & $1.5 \pm$ & & 0.14 & \\
\hline Age 6-9 & $1.5 \pm 0.5$ & $1.7 \pm$ & & 0.15 & \\
\hline
\end{tabular}

${ }^{a}$ Mean $d_{2-3} f s(S D)$ is per tooth. Some teeth were excluded due to presence of sealants. ${ }^{b} p$ value from $\chi^{2}$ test. ${ }^{c} p$ value from $t$ test. 
Table 2. Bivariate associations between dental caries and enamel hypoplasia of primary second molars

\begin{tabular}{|c|c|c|c|c|c|c|c|c|c|c|}
\hline \multirow[t]{2}{*}{ Person level (per person) } & \multirow[t]{2}{*}{$\mathrm{n}$} & \multirow{2}{*}{$\begin{array}{l}\mathrm{d}_{2-3} \mathrm{f} \\
\text { prevalence }\end{array}$} & \multirow{2}{*}{$\begin{array}{l}\text { Relative risk } \\
(95 \% \text { CI) }\end{array}$} & \multirow{2}{*}{$\begin{array}{l}\mathrm{p} \\
\text { value }^{\mathrm{a}}\end{array}$} & \multicolumn{5}{|c|}{$\mathrm{d}_{2-3}$ fs count } & \multirow{2}{*}{$\begin{array}{l}\mathrm{p} \\
\text { value }^{\mathrm{b}}\end{array}$} \\
\hline & & & & & 0 & 1 & 2 & 3 & $4+$ & \\
\hline \multicolumn{11}{|l|}{ Caries prevalence at age 5} \\
\hline Without hypoplasia & 472 & $16.9 \%$ & reference & \multirow[t]{2}{*}{0.03} & 392 & 29 & 19 & 12 & 20 & \multirow[t]{2}{*}{0.03} \\
\hline With hypoplasia & 19 & $36.8 \%$ & $2.17(1.17$ to 4.05$)$ & & 12 & 3 & 1 & 1 & 2 & \\
\hline \multicolumn{11}{|l|}{ Caries prevalence at age 9} \\
\hline Without hypoplasia & 469 & $34.5 \%$ & reference & \multirow[t]{2}{*}{0.11} & 307 & 36 & 37 & 19 & 70 & \multirow[t]{2}{*}{0.07} \\
\hline With hypoplasia & 19 & $52.6 \%$ & $1.52(0.98$ to 2.38$)$ & & 9 & 1 & 2 & 1 & 6 & \\
\hline \multicolumn{11}{|l|}{ Caries increment (age 5-9) } \\
\hline Without hypoplasia & 469 & $30.5 \%$ & reference & \multirow[t]{2}{*}{0.12} & 326 & 39 & 30 & 21 & 53 & \multirow[t]{2}{*}{0.15} \\
\hline With hypoplasia & 19 & $47.4 \%$ & $1.55(0.95$ to 2.54$)$ & & 10 & 3 & 3 & 0 & 3 & \\
\hline Tooth level (per tooth) & $\mathrm{n}$ & $\begin{array}{l}\mathrm{d}_{2-3} \mathrm{f} \\
\text { prevalence }\end{array}$ & $\begin{array}{l}\text { Relative risk } \\
(95 \% \text { CI })\end{array}$ & $\begin{array}{l}\mathrm{p} \\
\text { value }^{\mathrm{c}}\end{array}$ & Estin & ated & ${ }_{2-3} \mathrm{fs}^{\mathrm{d}}$ & $(95 \%$ & & $\begin{array}{l}\mathrm{p} \\
\text { value }^{\mathrm{d}}\end{array}$ \\
\hline \multicolumn{11}{|l|}{ Caries prevalence at age 5} \\
\hline Without hypoplasia & 1,849 & $7.6 \%$ & reference & \multirow[t]{2}{*}{$<0.01$} & 0.11 & .08 & $0.14)$ & & & \multirow[t]{2}{*}{$<0.01$} \\
\hline With hypoplasia & 32 & $28.1 \%$ & $3.11(1.69$ to 5.73$)$ & & 0.40 & .21 & $0.75)$ & & & \\
\hline \multicolumn{11}{|l|}{ Caries prevalence at age 9} \\
\hline Without hypoplasia & 1,831 & $18.3 \%$ & reference & \multirow[t]{2}{*}{0.02} & 0.34 & .29 & $0.41)$ & & & \multirow[t]{2}{*}{$<0.01$} \\
\hline With hypoplasia & 31 & $41.9 \%$ & $1.75(1.11$ to 2.74$)$ & & 0.76 & 0.46 & $1.26)$ & & & \\
\hline \multicolumn{11}{|l|}{ Caries increment (age 5-9) } \\
\hline Without hypoplasia & 1,831 & $14.4 \%$ & reference & \multirow[t]{2}{*}{0.16} & 0.25 & .21 & $0.30)$ & & & \multirow[t]{2}{*}{0.06} \\
\hline With hypoplasia & 31 & $25.8 \%$ & $1.55(0.84$ to 2.85$)$ & & 0.46 & .25 & $0.87)$ & & & \\
\hline
\end{tabular}

notes to tables 3 and 4, and were used in backward elimination GEE procedures.

Final GEE models for caries presence/incidence and counts/increments included all variables that remained jointly significant $(\mathrm{p}<0.05)$, plus hypoplasia, and are detailed in tables 3 and 4 , respectively. The tooth level data $(n=1,892)$ needed to compensate for intrasubject correlation and hence the tooth-level multivariable models used GEE methods. Logistic GEE models predicting caries (yes/no) at age 5 and 9 , and any new caries from age 5 to 9, were fitted using the aforementioned selected predictors, along with hypoplasia, and final models are presented in table 3. The models were developed for repeatedmeasures or clustered data. After controlling for the other important predictor variables, children with enamel hypoplasia had a significantly higher risk of caries (see 'between-subject' hypoplasia variable) at both age 5 (odds ratio, $\mathrm{OR}=7.56, \mathrm{p}<0.01)$ and age $9(\mathrm{OR}=5.16, \mathrm{p}=0.03)$, but no significant association with caries incidence (OR = $1.02, \mathrm{p}=0.97)$. Within-subject hypoplasia was not a sig- nificant factor in any of the models, indicating that, for a given subject, the caries risk for hypoplastic and nonhypoplastic teeth was comparable.

Repeated-measures negative binomial GEE models were constructed to predict the number of decayed and filled surfaces $\left(\mathrm{d}_{2-3} \mathrm{fs}\right)$ at age 5 , age 9 and age $5-9 \mathrm{~d}_{2-3} \mathrm{fs}$ increment (table 4). Between-subject hypoplasia was a statistically significant predictor for $\mathrm{d}_{2-3} \mathrm{fs}$ at age 5 (parameter estimate $=1.89, \mathrm{p}<0.01$ ), age 9 (parameter estimate $=1.39, \mathrm{p}<0.01$ ), and age 5-9 caries increment (parameter estimate $=1.28, \mathrm{p}=0.04$ ). Within subjects, there was a significant increase in age $5 \mathrm{~d}_{2-3}$ fs associated with teeth exhibiting hypoplasia (parameter estimate $=1.46$, $\mathrm{p}=0.03$ ) and age $9 \mathrm{~d}_{2-3} \mathrm{fs}$ (parameter estimate $=0.90, \mathrm{p}=$ 0.03 ), but not age 5-9 $\mathrm{d}_{2-3}$ fs increment (parameter estimate $=0.70, p=0.19$ ). It is noteworthy that all $\mathrm{d}_{2-3}$ fs estimates were higher, both for subjects with more hypoplasia and for teeth exhibiting hypoplasia throughout the analyses, even though some were not statistically significant. 
Table 3. Tooth level logistic GEE models for caries (yes/no) using backward elimination method

\begin{tabular}{|c|c|c|c|c|}
\hline Tooth outcome & Predictor variables & Odds ratio unit ${ }^{\mathrm{a}}$ & $\begin{array}{l}\text { Odds ratio } \\
(95 \% \mathrm{CI})\end{array}$ & $\begin{array}{l}\mathrm{p} \\
\text { value }\end{array}$ \\
\hline \multirow{5}{*}{$\begin{array}{l}\text { Caries presence } \\
\text { (yes/no) at age } 5^{c}\end{array}$} & between-subject hypoplasia ${ }^{b}$ & all second molars (vs. none) & $7.56(2.28-25.07)$ & $<0.01$ \\
\hline & within-subject hypoplasiab & 1 tooth more than subject average & $2.80(0.88-8.95)$ & 0.08 \\
\hline & dental exam age, years & 1 year & $1.91(1.11-3.28)$ & 0.02 \\
\hline & breast-feeding & $<6$ months (vs. $\geq 6$ months) & $2.15(1.16-3.98)$ & 0.01 \\
\hline & average home tap water fluoride concentration ( $3-5$ years of age) & $1.0 \mathrm{ppm}$ & $2.36(1.29-4.30)$ & $<0.01$ \\
\hline \multirow{4}{*}{$\begin{array}{l}\text { Caries presence } \\
\left(\text { yes/no) at age } 9^{d}\right.\end{array}$} & between-subject hypoplasia ${ }^{b}$ & all second molars (vs. none) & $5.16(1.15-23.14)$ & 0.03 \\
\hline & within-subject hypoplasia ${ }^{\mathrm{b}}$ & 1 tooth more than subject average & $1.61(0.68-3.81)$ & 0.27 \\
\hline & average daily fluoride intake during 5-9 years of age & $1 \mathrm{mg} F$ per day & $1.90(1.05-3.42)$ & 0.03 \\
\hline & average daily toothbrushing frequency during $5-9$ years old & one more time per day & $2.16(1.49-3.09)$ & $<0.01$ \\
\hline \multirow{5}{*}{$\begin{array}{l}\text { Caries incidence } \\
\text { (yes/no) } \\
\text { age } 5-9^{e}\end{array}$} & between-subject hypoplasia ${ }^{\mathrm{b}}$ & all second molars (vs. none) & $1.02(0.31-3.39)$ & 0.97 \\
\hline & within-subject hypoplasia ${ }^{\mathrm{b}}$ & 1 tooth more than subject average & $1.73(0.40-7.57)$ & 0.47 \\
\hline & previous caries (age 5) & $1 \mathrm{~d}_{2-3} \mathrm{fs}$ & $5.08(3.48-7.42)$ & $<0.01$ \\
\hline & average daily fluoride intake during 5-9 years of age & $1 \mathrm{mg} F$ per day & $1.90(1.01-3.59)$ & 0.048 \\
\hline & average daily toothbrushing frequency during $5-9$ years old & one more time per day & $1.97(1.36-2.85)$ & $<0.01$ \\
\hline
\end{tabular}

\footnotetext{
a Odds ratios represent the change in odds relative to 1 unit change in the predictor variable.

b The 'between-subject' hypoplasia variable addresses differences between subjects with and without hypoplasia, and the 'within-subject' hypoplasia variable addresses differences within subjects (for subjects having hypoplasia) between teeth with hypoplasia and teeth without hypoplasia.

${ }^{\mathrm{c}}$ Additional variables included for consideration were: age 3-5 averages for estimated daily fluoride intake (mg F), estimated soda pop intake (oz) and daily tooth brushing frequency.

d One additional variable was included for consideration: age 5-9 average estimated soda pop intake (oz).

${ }^{e}$ Additional variables included for consideration were: previous (age 5) $\mathrm{d}_{2-3} \mathrm{fs}$, and age 5-9 average estimated soda pop intake (oz).
}

Table 4. Tooth level negative binomial GEE models of caries $\mathrm{d}_{2-3}$ fs counts and important predictor variables using backward elimination

\begin{tabular}{|c|c|c|c|c|}
\hline Outcome & Predictor variables & Unit $^{\mathrm{a}}$ & $\begin{array}{l}\text { Parameter estimate } \\
(95 \% \mathrm{CI})\end{array}$ & $\begin{array}{l}\mathrm{p} \\
\text { value }\end{array}$ \\
\hline $\begin{array}{l}\text { Number of decayed } \\
\text { or filled surfaces at } \\
\text { age } 5\left(d_{2-3} f s\right)^{c}\end{array}$ & $\begin{array}{l}\text { between-subject hypoplasia }^{\mathrm{b}} \\
\text { within-subject hypoplasia }{ }^{\mathrm{b}} \\
\text { age at dental examination, years } \\
\text { average home tap water fluoride concentration ( } 3-5 \text { years of age) } \\
\text { average daily soda pop intake during } 3-5 \text { years of age }\end{array}$ & $\begin{array}{l}\text { all second molars (vs. none) } \\
1 \text { tooth more than subject average } \\
1 \text { year } \\
1 \mathrm{ppm} \\
1 \mathrm{oz}\end{array}$ & $\begin{array}{l}1.89(0.72-3.06) \\
1.46(0.14-2.79) \\
0.62(0.22-1.03) \\
1.09(0.51-1.68) \\
0.08(0.01-0.15)\end{array}$ & $\begin{array}{r}<0.01 \\
0.03 \\
<0.01 \\
<0.01 \\
0.03\end{array}$ \\
\hline $\begin{array}{l}\text { Number of decayed } \\
\text { or filled surfaces } \\
\text { (dfs) at age } 9^{d}\end{array}$ & $\begin{array}{l}\text { between-subject hypoplasia }^{\mathrm{b}} \\
\text { within-subject hypoplasia } \\
\text { age at dental examination, years } \\
\text { average daily fluoride intake during 5-9 years of age } \\
\text { average daily toothbrushing frequency during 5-9 years old }\end{array}$ & $\begin{array}{l}\text { all second molars (vs. none) } \\
1 \text { tooth more than subject average } \\
1 \text { year } \\
1 \text { mg F per day } \\
\text { one more time per day }\end{array}$ & $\begin{array}{l}1.39(0.50-2.29) \\
0.90(0.12-1.68) \\
0.27(0.04-0.50) \\
0.65(0.08-1.23) \\
0.70(0.34-1.06)\end{array}$ & $\begin{array}{r}<0.01 \\
0.03 \\
0.03 \\
0.03 \\
<0.01\end{array}$ \\
\hline $\begin{array}{l}d_{2-3} f \text { increment } \\
\text { (number of new } \\
d_{2-3} f s \text { ) age } 5-9^{e}\end{array}$ & $\begin{array}{l}\text { between-subject hypoplasia }^{\text {b }} \\
\text { within-subject hypoplasia } \\
\text { age } 5 d_{2-3} \text { fs } \\
\text { average daily toothbrushing frequency during } 5-9 \text { years old }\end{array}$ & $\begin{array}{l}\text { all second molars (vs. none) } \\
1 \text { tooth more than subject average } \\
1 \mathrm{~d}_{2-3} \mathrm{fs} \\
\text { one more time per day }\end{array}$ & $\begin{array}{l}1.28(0.11-2.45) \\
0.70(0.33-1.74) \\
0.14(0.11-0.17) \\
0.72(0.38-1.06)\end{array}$ & $\begin{array}{r}0.04 \\
0.19 \\
<0.01 \\
<0.01\end{array}$ \\
\hline
\end{tabular}

\footnotetext{
${ }^{a}$ The parameter estimate represents the change in estimated $\mathrm{d}_{2-3}$ fs relative to 1 unit change in the predictor variable.

b The 'between-subject' hypoplasia variable addresses differences between subjects with and without hypoplasia, and the 'within-subject' hypoplasia variable addresses differences within subjects (for subjects having hypoplasia) between teeth with hypoplasia and teeth without hypoplasia.

${ }^{c}$ One additional variable was included for consideration: breast-fed 6 months or more (yes/no).

d Additional variables included for consideration were: sex, SES, and age 5-9 average estimated soda pop intake (oz).

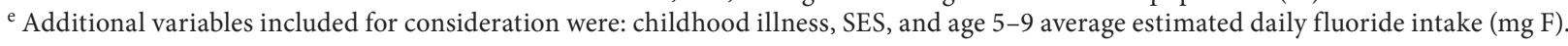




\section{Discussion}

This prospective study examined the risk of dental caries on primary second molars associated with enamel hypoplasia in a cohort of children from birth to age 9 . The primary second molars were the teeth most consistently present at both examinations, and limiting to this tooth type helped to reduce the confounding effects of differential caries rates on different tooth types (canines had much lower caries rates). Therefore, although some of the total caries experience would be missed due to many primary teeth not being included, the confounding effect of differing caries rates would be avoided.

The results of the bivariate analyses indicated that enamel hypoplasia was significantly associated with dental caries. The trend was generally consistent at all levels of bivariate analysis, at both the person and tooth levels, for age 5 findings, and to a lesser degree for age 9 and age 5-9 incidence. However, results were not consistently significant. Theoretically, from the view of pathogenesis, teeth with enamel hypoplasia should have a higher risk of dental caries because hypoplastic enamel surfaces have reduced thickness of enamel and may provide a more suitable local environment for adhesion and colonization of cariogenic bacteria [Li et al., 1996], and are more acidsoluble [Zheng et al., 1998].

The results were somewhat consistent with previous studies, which suggested that enamel defects increase the risk of dental caries in the affected teeth [Pascoe and Seow, 1994; Li et al., 1996; Lai et al., 1997; Milgrom et al., 2000; Montero et al., 2003; Oliveira et al., 2006]. From the results of multivariable regression analyses, the between-subject effect from enamel hypoplasia was consistently significant except for age 5-9 incidence (table 3). This indicates that subjects with hypoplasia are more at risk for caries at age 5 and 9 , and also likely to have more surfaces affected by caries (table 4). It should be noted, however, that most of the caries in the study was treated (i.e. 'filled'), so that some dentists' decisions to preferentially treat hypoplastic teeth at an earlier age may also partially explain these findings.

However, the within-subject effect from enamel hypoplasia was not consistently significant. This might suggest that, for subjects exhibiting enamel hypoplasia, the risk for developing caries is similar for all teeth. Also, the weaker effects of hypoplasia on caries increments may indicate that hypoplasia has a stronger early influence (seen in age 5 caries experience, with a carryover effect on age 9 caries experience), but that the effect may not continue to put subjects at an increased risk for caries many years after eruption.

Enamel Hypoplasia and Caries Experience of Primary Second Molars
Despite the dramatic decline in dental caries experience in the last several decades in the US and some other developed countries, dental caries remains a significant health problem [National Institute of Health, 2000]. Extensive evidence indicates that dental caries is a communicable infectious disease [Caufield, 2005], which involves many environmental and host factors and the interactions among these factors [Featherstone, 1990, 2000; Fejerskov and Kidd, 2003]. This supports a paradigm shift from a surgical model which focuses mainly on removing the carious tooth structure followed by replacement with a restorative material to a biological/medical model in which the individual's overall and site-specific susceptibility are assessed and interventions can be individualized to prevent and control this infection [Horowitz, 2004]. Therefore, in contemporary management of dental caries with this medical model, caries risk assessment plays a crucial role [Powell, 1998; Reich et al., 1999; Hausen, 2003; Fontana and Zero, 2006; American Dental Association, 2009]. Unfortunately, there is no simple, valid, and reliable caries risk assessment model currently available. Previous caries experience and/or untreated (active) lesions are the best predictors for future caries development [Bibby and Shern, 1978; Disney et al., 1992; Leverett et al., 1993; Hausen et al., 1994; Vanobbergen et al., 2001; Featherstone et al., 2003]. However, these predictors are post-disease and do not serve well for early detection and primary prevention of the disease. As a result, they might not be as useful to a practitioner trying to control and prevent caries at the earliest stages in susceptible patients. In this study, after controlling for other potential factors, multivariable regression models suggested that enamel hypoplasia seems to be a good predictor. Enamel hypoplasia might not only itself render the tooth more prone to caries development, but also be in part a proxy indicator for substandard nutrition and/or childhood infection. All of these negative early life experiences during enamel formation might manifest collectively as enamel hypoplasia. These sorts of events will put a child at higher risk for dental caries. Together with other information, such as fluoride concentration of home water source, other fluoride exposures, soda pop intake, and brushing frequency, a risk assessment model that considers enamel hypoplasia data could provide good prediction of early caries risk, particularly at the tooth level. Enamel hypoplasia might have good potential to serve as a predictor of caries experience, because the teeth could be examined for hypoplasia right after eruption, before carious lesions develop. Thus, early intervention measures could be applied with at-risk children to prevent early caries stages.

Caries Res 2009:43:345-353 
The results from this study indicate that enamel hypoplasia is a significant predictor for dental caries of childhood. However, several limitations of the study must be recognized. Several important factors related to dental caries, such as oral bacteria and plaque levels, were not included in the analysis. The subjects were mostly Caucasian and from families with relatively high SES in Iowa. Such a convenience sample limits the generalizability of the results to other groups of children. In addition, the number of children who were diagnosed with hypoplasia (only 19) was small and a small change in diagnosis reliability could have an impact on the results. Within the study limitations, this prospective, longitudinal study of the birth cohort provided evidence that enamel hypopla- sia could be a clinically meaningful predictor for dental caries. More studies are needed to better understand the role of enamel hypoplasia in caries development, including studies of longer duration, with larger, more diverse samples, and assessing other important factors related to dental caries. Also, studies are needed to understand the factors related to the time-dependent effects of enamel hypoplasia on caries presence at age 5 and 9 .

\section{Acknowledgments}

This study was supported in part by NIH R01-DE09551, R01DE12101, and M01-RR00059.

\section{References}

American Dental Association: Caries risk assessment classification. http://www.ada.org/ prof/resources/topics/topics_caries_instructions.pdf. Accessed March 2009.

Bibby BG, Shern RJ (eds): Methods of Caries Prediction. Proceedings of a Workshop Conference. Washington, Information Retrieval, 1978.

Caufield PW: Dental caries: an infectious and transmissible disease: where have we been and where are we going? NY State Dent J 2005;71:23-27.

Daneshkazemi AR, Davari A: Assessment of DMFT and enamel hypoplasia among junior high school children in Iran. J Contemp Dent Pract 2005;6:85-92.

Disney JA, Graves RC, Stamm JW, Bohannan HM, Abernathy JR, Zack DD: The University of North Carolina Caries Risk Assessment study: further developments in caries risk prediction. Community Dent Oral Epidemiol 1992;20:64-75.

-Ellwood RP, O’Mullane D: The association between developmental enamel defects and caries in populations with and without fluoride in their drinking water. J Public Health Dent 1996;56:76-80.

Evans S, Li L: A comparison of goodness of fit tests for the logistic GEE model. Stat Med 2005;24:1245-1261.

Featherstone JD: An updated understanding of the mechanism of dental decay and its prevention. Nutr Q 1990;14:5-11.

Featherstone JD: The science and practice of caries prevention. J Am Dent Assoc 2000;131: 887-899.
Featherstone JD, Adair SM, Anderson MH, Berkowitz RJ, Bird WF, Crall JJ, Den Besten PK, Donly KJ, Glassman P, Milgrom P, Roth JR, Snow R, Stewart RE: Caries management by risk assessment: consensus statement, April 2002. J Calif Dent Assoc 2003;31:257269.

Fejerskov O, Kidd EAM: Clinical cariology and operative dentistry in the twenty-first century; in Fejerskov O, Kidd EAM (eds): Dental Caries, the Disease and Clinical Management. Oxford, Blackwell Munksgaard, 2003, pp 3-7.

Fontana M, Zero DT: Assessing patients' caries risk. J Am Dent Assoc 2006;137:1231-1239.

Hausen H: Caries prediction; in Fejerskov O, Kidd EAM (eds): Dental Caries, the Disease and Clinical Management. Oxford, Blackwell Munksgaard, 2003, pp 327-339.

Hausen H, Seppä L, Fejerskov O: Can caries be predicted?; in Thylstrup A, Fejerskov O (eds): Textbook of Clinical Cariology, ed 2. Copenhagen, Munksgaard, 1994, pp 393-411.

Hong L, Levy SM, Warren JJ, Bergus GR, Dawson DV, Wefel JS: Primary tooth fluorosis and amoxicillin use during infancy. J Public Health Dent 2004;64:38-44.

Horowitz AM: A report on the NIH consensus development conference on diagnosis and management of dental caries throughout life. J Dent Res 2004;83:C15-C17.

Ismail AI, Brodeur JM, Gagnon P, Payette M, Picard D, Hamalian T, Olivier M, Eastwood BJ: Prevalence of noncavitated and cavitated carious lesions in a random sample of 7-9year-old schoolchildren in Montreal. Community Dent Oral Epidemiol 1992;20:250 255.
Kanchanakamol U, Tuongratanaphan S, Tuongratanaphan S, Lertpoonvilaikul W, Chittaisong C, Pattanaporn K, Navia JM, Davies GN: Prevalence of developmental enamel defects and dental caries on rural preschool Thai children. Community Dent Health 1996;13:204-207.

Lai PY, Seow WK, Tudehope DI, Rogers Y: Enamel hypoplasia and dental caries in verylow birthweight children: a case controlled, longitudinal study. Pediatr Dent 1997;19:4249.

Leverett DH, Proskin HM, Featherstone JD: Caries risk assessment in a longitudinal discrimination study. J Dent Res 1993;72:538543.

Levy SM, Kiritsy MC, Slager SL, Warren JJ: Patterns of dietary fluoride supplement use during infancy. J Public Health Dent 1998;58: 228-233.

Levy SM, Kiritsy MC, Slager SL, Warren JJ, Kohout FJ: Patterns of fluoride dentifrice use among infants. Pediatr Dent 1997;19:50-55.

Li Y, Navia JM, Bian JY: Caries experience in deciduous dentition of rural Chinese children 3-5 years old in relation to the presence or absence of enamel hypoplasia. Caries Res 1996;30:8-15.

Mancl LA, Leroux BG, DeRouen TA: Betweensubject and within-subject statistical information in dental research. J Dent Res 2000; 79:1778-1781.

Matee MIN, Van't Hof MA, Maselle SY, Mikx FHM, van Palenstein Herlderman WH: Nursing caries, linear hypoplasia, and nursing and weaning habits in Tanzanian infants. Community Dent Oral Epidemiol 1994;22:289-293. 
Milgrom P, Riedy CA, Weinstein P, Tanner AC, Manibusan LB: Dental caries and its relationship to bacterial infection hypoplasia, diet and oral hygiene in 6-to 36-month-old children. Community Dent Oral Epidemiol 2000;28:295-306.

Montero MJ, Douglass JM, Mathieu GM: Prevalence of dental caries and enamel defects in Connecticut Head Start children. Pediatr Dent 2003;25:235-239.

National Institute of Health, US Department of Health and Human Services: Oral Health in America: A Report of the Surgeon General. Rockville, National Institute of Dental and Craniofacial Research, National Institute of Health, 2000.

Oliveira AFB, Chaves AMB, Rosenblatt A: The influence of enamel defects on the development of early childhood caries in a population with low socioeconomic status: a longitudinal study. Caries Res 2006;40:296-302.

-Pascoe L, Seow WK: Enamel hypoplasia and dental caries in Australian Aboriginal children: prevalence and correlation between the two diseases. Pediatr Dent 1994;16:193-199.
Pendrys DG: The Fluorosis Risk Index: a method for investigating risk factors. J Public Health Dent 1990;50:291-299.

Pitts NB: Diagnostic tools and measurements impact on appropriate care. Community Dent Oral Epidemiol 1997;25:24-35.

Pitts NB, Fyffe HE: The effect of varying diagnostic thresholds upon clinical caries data for a low prevalence group. J Dent Res 1988; 67:592-596.

Powell V: Caries risk assessment: relevance to the practitioner. J Am Dent Assoc 1998;129: 349-353.

Reich E, Lussi A, Newbrun E: Caries risk assessment. Int Dent J 1999;49:15-26.

Russell AL: The differential diagnosis of fluoride and non-fluoride enamel opacities. J Public Health Dent 1961;21:143-146.

Seow WK: Enamel hypoplasia in the primary dentition: a review. J Dent Child 1991;58 441-452.

Shenkin JD, Broffitt B, Levy SM, Warren JJ: The association between environmental tobacco smoke and primary tooth caries. J Public Health Dent 2004;64:184-186.
-Slayton RL, Warren JJ, Kanellis MJ, Levy SM, Islam M: Prevalence of enamel hypoplasia and isolated opacities in the primary dentition. Pediatr Dent 2001;23:32-36.

Suckling GW: Development defects of enamel historical and present-day perspectives of their pathogenesis. Adv Dent Res 1989;3:8794.

Vanobbergen J, Martens L, Lesaffre E, Bogaerts K, Declerck D: The value of a baseline caries risk assessment model in the primary dentition for the prediction of caries incidence in the permanent dentition. Caries Res 2001; 35:442-450.

-Warren JJ, Levy SM, Kanellis MJ: Dental caries in the primary dentition: assessing prevalence of cavitated and non-cavitated lesions. J Public Health Dent 2002;62:109-114.

ZZheng S, Deng H, Gao X: Studies on developmental enamel defects in the primary dentition of children with histories of low birth weight and prematurity and their susceptibility to dental caries. Zhonghua Kou Qiang Yi Xue Za Zhi 1998;33:270-272. 